\title{
Growth and characterization of an organic NLO crystal:4-chloro- 4-methyl benzylidene aniline
}

\author{
L. Jothi ${ }^{1}$ and K. Ramamurthi ${ }^{2}$ \\ ${ }^{1}$ Department of Physics, N.K.R Government Arts College for Women, Namakkal-637001, Tamilnadu, India \\ ${ }^{2}$ Crystal Growth \& Thin Film Lab., Dept. of Physics, Bharathidasan University, Tiruchirappalli-620024, Tamilnadu, India \\ jothilakshman@gmail.com
}

\begin{abstract}
In this paper, we report for the first time the synthesis, growth and characterization of a new organic NLO single crystal of 4 choloro-4-methyl benzylidene aniline. The title compound is synthesized and single crystals were grown by the slow evaporation technique at constant temperature. The grown single crystals have been characterized by single crystal XRD; powder XRD, FTIR, NMR, UV-Vis, thermal analysis and microhardness studies. From the XRD study, it is found that the crystals are in the monoclinic system. The FTIR spectrum was recorded to identify the various functional groups present in the group compound. The formation of the product was confirmed from NMR spectral analysis. The optical transparency was determined by UV-visible spectroscopy. The melting point is found to be $108^{\circ} \mathrm{C}$ from the DSC curve. The second harmonic generation (SHG) efficiency of the crystal was obtained using ND-YAG laser which is found to be 7.5 times that of KDP.
\end{abstract}

Keywords: Single crystal growth, NLO crystal, XRD data

Materials with large nonlinear optical susceptibilities are of current interest in the area of harmonic generation and optical modulation. In recent years, some polar organic crystals, which form a non-centrosymmetric structure exhibit second-order nonlinear optical properties that far surpassed those of the conventional materials has led to the synthesis and evaluation of a wide range of potentially useful solids (Prasad \& Willams, 1991) materials showing high optical non linearity have potential applications in signal transmission, data storage, optical switching, laser printing, displays, inflorescence, photolithography, remote sensing, chemical and biological species detection, high resolution spectroscopy, medical diagnosis and underwater monitoring \& communication (Chemla \& Zyss, 1987). Organic molecules with significant nonlinear optical activity generally consist of a $\Pi$ - electron conjugated structure. The conjugation $\pi$-electron moiety provides a pathway for the entire length of conjugation under the perturbation of an external electric field. Fictionalization of both ends of the $n$ bond systems with appropriate electron donor and acceptor group can increase the asymmetric electronic distribution in either or both the ground and excited states, thus leading to an increased optical nonlinearity (Bailey et al.,1993; Lakshmana Perumal et al., 2002; Srinivasan et al., 2002; Yang et al., 2006). Among many organic compounds reported for the SHG benzylidene aniline derivates are noticeable materials for the excellent green light transmittance and good crystal stability (Shepherd et al., 1991; John \& Whittaker 2004; Nixon Azariah et al., 2004; Leela et al., 2009).

Scheme1. Molecular structure of CMBA

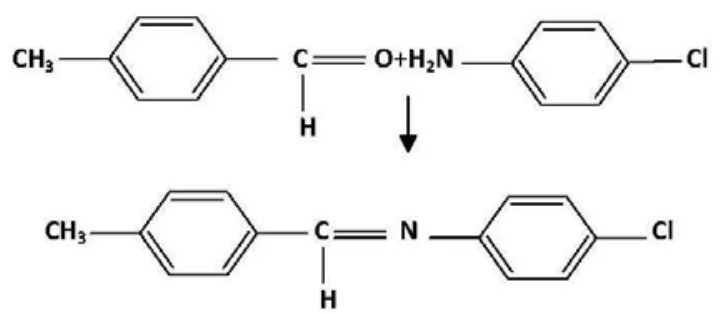

"Single crystal growth of organic NLO" http://www.indjst.org
In this work we introduce a new benzylidene aniline derivative: 4-chloro-4-methyl benzylidene aniline (CMBA). The molecular structure of CMBA is shown Scheme1. The grown crystals have been subjected to the characterizations like XRD, FTIR, NMR, UV-vis, thermal analysis, powder SHG and micro-hardness studies.

\section{Materials and methods}

\section{Synthesis and purification of CMBA}

CMBA raw material was synthesised from p-methyl benzaldehyde and p-chloro aniline by condensation. High purity chemicals from Merck (Germany) were used for this synthesis. The synthesized CMBA was further purified by step-by-step recrystallization process. The high purity of the material was confirmed by thermo gravimetric analysis. The melting point of the grown crystal is found to be $108^{\circ} \mathrm{C}$. The chemical reaction is shown in Scheme 1.

Crystal growth

Crystal growth experiments were performed by slow evaporation method. The following procedure was adopted for the growth by slow evaporation. Saturated solutions of $\mathrm{CMBA}$ were prepared at $35^{\circ} \mathrm{C}$ with reference to the solubility data. The solutions were heated $5^{\circ} \mathrm{C}$ above their saturation temperature to remove any undissolved crystallites. The crystallizer was then placed in the constant temperature bath and cooled to the predefined saturation temperature, $\quad 35^{\circ} \mathrm{C}$. Few perforations were made for the evaporation of solvent. On the fourth day, nucleation was noticed and it was allowed to grow for a period of 30 days. The harvested crystal has the dimension $8 \mathrm{~mm} \times 5 \mathrm{~mm} \times 1 \mathrm{~mm}$. The photograph of the grown Indian J.Sci.Technol. 
crystal is shown Fig.1.

Results and discussion

Single crystal $X$-ray diffraction

The single crystal X-ray

diffraction is recorded with the

Bruker Smart Apex instrument and the wavelength of $\mathrm{X}$-ray used was $0.7093 \mathrm{~A}^{\circ}$ (Target-molybdenum). From single crystal XRD the lattice parameters were calculated and this crystal belongs to monoclinic system. The lattice parameters calculated from single crystal XRD are $a=5.964(2) A^{\circ}, b=7.415(2)$ $A^{\circ}, c=13.693(3) A^{\circ}$ and $\alpha=$

Fig. 1. Photograph of the CMBA grown crystal

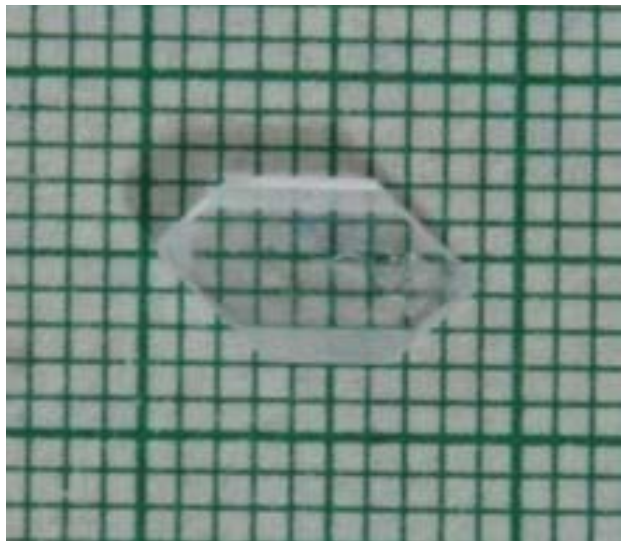

ISSN: 0974- 6846

Vol. 4 No. 6 (June 2011)

group assignments are shown in
Table 1 which confirms the chemical structure of the synthesized compound.

NMR analysis

The commonly used NMR spectroscopy techniques are notably ${ }^{1} \mathrm{H}$ NMR and ${ }^{13} \mathrm{C}$ NMR. Carbon is the core element in organic chemistry and hence ${ }^{13} \mathrm{C}$ NMR plays an important role in determining the structure of unknown organic molecules and the study of organic reactions and processes. In the present study, ${ }^{13} \mathrm{C}$ $90.00(0)^{\circ}, \quad \beta=99.37(2)^{\circ}, \quad Y=90.00(0)^{\circ}$. The other parameter calculated from single crystal $X R D$ is $V=$ 599.4(3) $\hat{A}^{3}$.

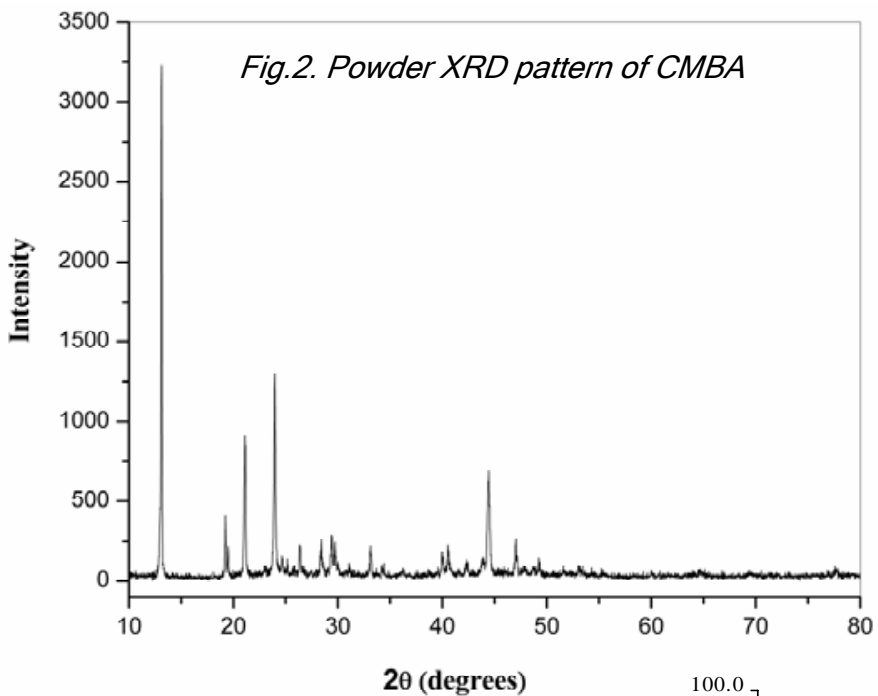

NMR spectral analysis was made on the powder form of the $\mathrm{p}$-CMBA crystal dissolved in deuterated organic solvent $\mathrm{CDCl}_{3}$ and recorded using AM $\times 400 \mathrm{NMR}$ analyzer at $27^{\circ} \mathrm{C}$. The ${ }^{13} \mathrm{C}$ NMR spectrum of $p$-CMBA is shown in Fig.4. The alkyl methyl carbon produces its characteristics signal at $22.56 \mathrm{ppm}$. The signal at 77.93 $\mathrm{ppm}$ is due to $\mathrm{CDCl}_{3}$. The ester carbonyl carbon produces it's in the extreme down field region at $161.61 \mathrm{ppm}$. The remaining four peaks are due to the four types of carbon in the aromatic ring. Molecular structure of CMBA is thus confirmed from the NMR spectrum.

UV-Visible spectral analysis

UV-visible spectrum of the grown crystal was recorded using a SHIMADZU UV-VIS-NIR scanning spectrophotometer, model $3101 \mathrm{PC}$ (Fig.5 \& 6). There is no absorption of light from near UV to the near IR.

Absorption spectrum of the NLO material plays a major role in device fabrication. Wider the transparency window more will be the practical applicability of the

\section{Powder X-ray diffraction}

Powder X-ray diffraction studies were carried out to confirm the crystallinity using Rich Seifert X-ray diffractometer with the CuKa radiation in the range of $10^{\circ}-80^{\circ}$, insteps of $10^{\circ}$. The powder XRD pattern is shown in Fig.2. It reflects the good crystallinity of the grown crystal. The lattice parameters were calculated using TREOR programme, coincides with single crystal XRD results.

\section{FTIR studies}

The FTIR spectrum is recorded using Avatar 330 FT-IR thermo Nicolet spectrometer in the wavelength range $400-4000 \mathrm{~cm}^{-1}$ by $\mathrm{KBr}$ pellet technique. The middle infrared (IR) spectrum of the CMBA material is shown in Fig.3. The
Fig.3. FTIR Spectrum of CMBA

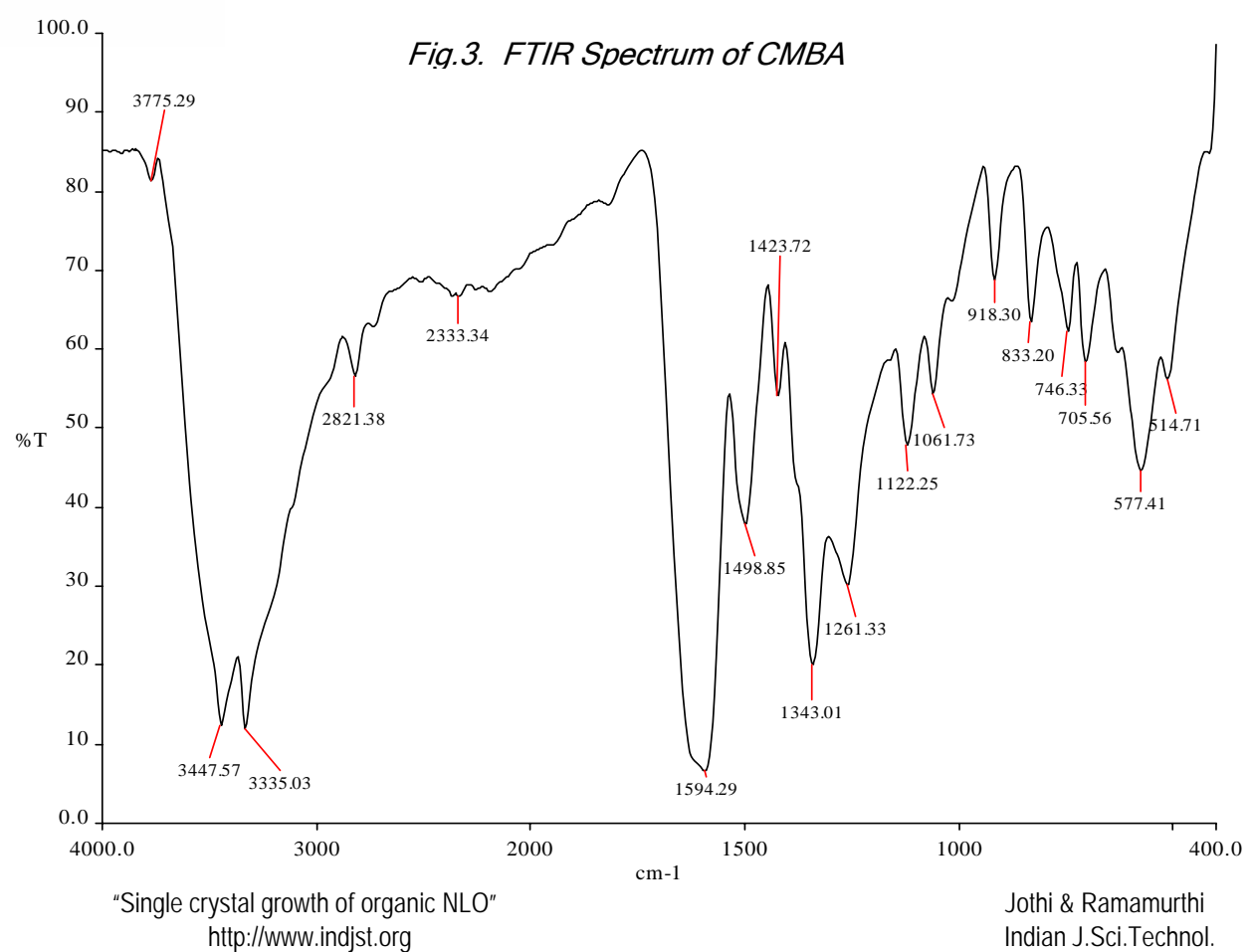


Fig. $4 .{ }^{13} \mathrm{C}$ NMR spectrum of CMBA

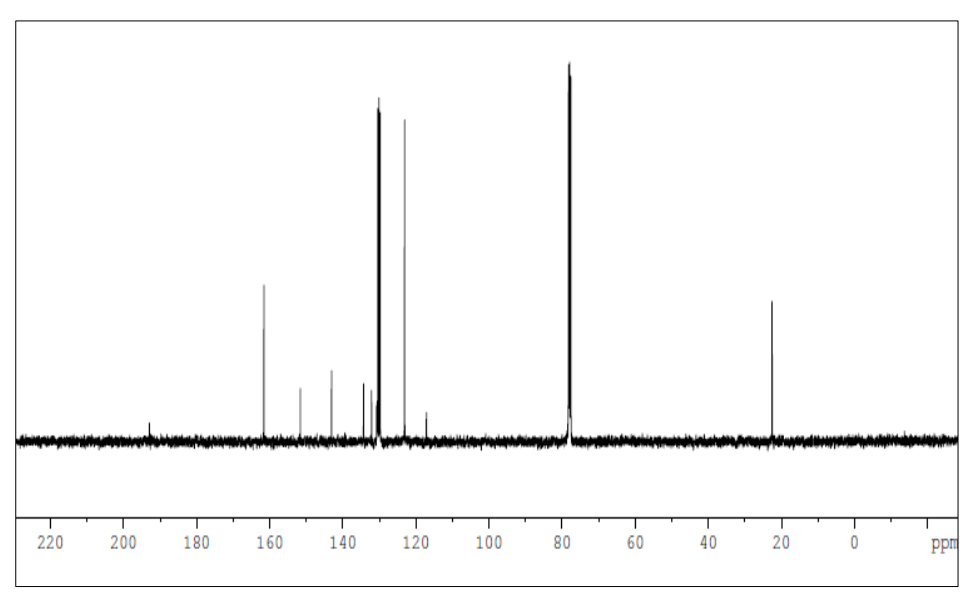

Table. 1. FTIR analysis

\begin{tabular}{|c|cc|}
\hline Peak $\left(\mathrm{cm}^{-1}\right)$ & \multicolumn{2}{|c|}{ Assignment } \\
\hline 3777.83 & O-H & Str \\
3437.03 & N-H & Str \\
2729.58 & O-H & Str \\
1710.44 & C=O & Str \\
1597.68 & C=C & Str \\
1475.14 & C-H & Def \\
1356.72 & C-N & Vibr \\
1225.72 & C-N & Vibr \\
1095.81 & C-OH & Str \\
1005.80 & C-H & Def \\
834.29 & C-H & Def \\
\hline
\end{tabular}
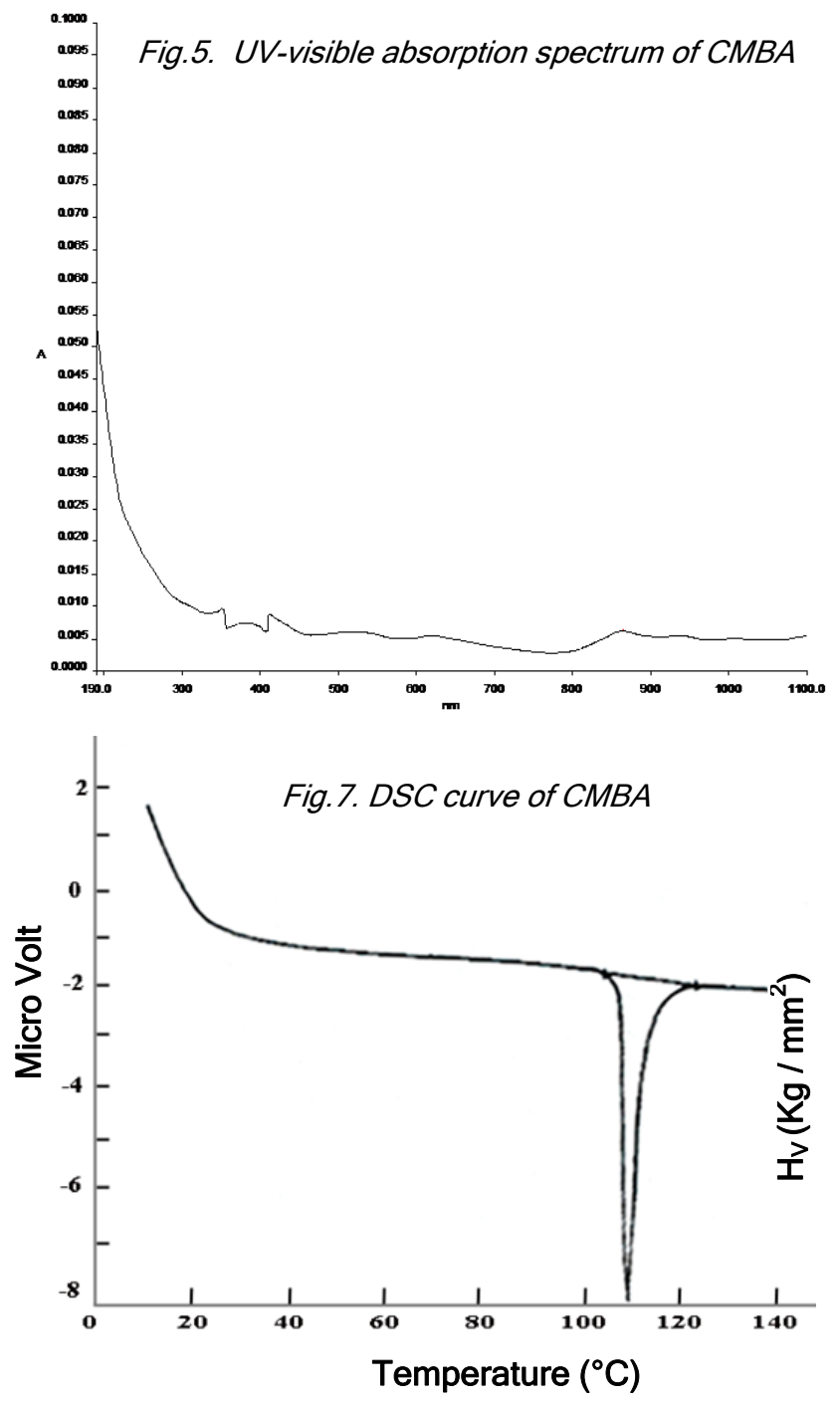

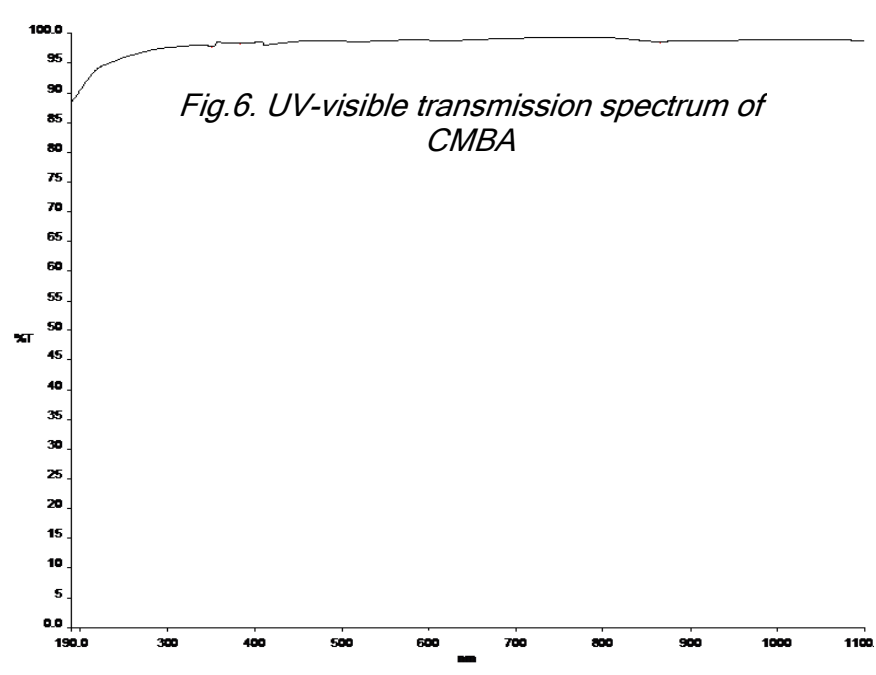

Fig.8. Hardness vs. Load for CMBA

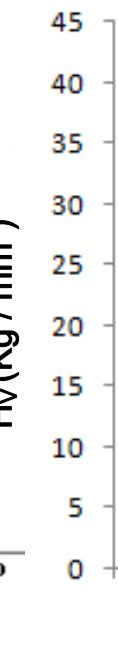

25

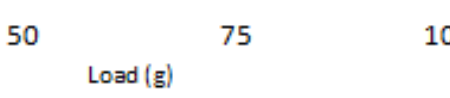
Indian J.Sci.Technol. 
material. Optical absorption spectrum of the grown crystal was recorded in the wave range of $1100-100 \mathrm{~nm}$. The absorption is negligible in the entire visible region of the electromagnetic spectrum. Therefore, it can be used as a potential material for SHG in the visible region.

\section{Thermal analysis}

Differential Scanning Calorimetry (DSC) was carried out for the CMBA crystals using a NET SZCH STA 409C. A characteristic curve is shown in Fig.7. A powder sample was used for the analysis in the temperature range from $0^{\circ} \mathrm{C}$ to $200^{\circ} \mathrm{C}$ with a heating rate of $10^{\circ} \mathrm{C} / \mathrm{min}$ in the nitrogen atmosphere. The crucible used was of alumina $\left(\mathrm{Al}_{2} \mathrm{O}_{3}\right)$, which served as a reference for the sample. In the DSC curve the sharp endothermic peak at $108^{\circ} \mathrm{C}$ is the melting point of CMBA. The sharpness of the peak confirms the good crystallinity of the synthesized compound.

\section{Second harmonic generation eficiency}

Second harmonic generation measurement was performed using Nd:YAG laser instrument, Model: DCR3G SPECTRA PHYSICS following Kurtz powder method. The crystal was ground into a fine powder and sandwiched between two quartz slides, which serve as sample cell. When Nd:YAG laser beam of wavelength $1.06 \mu \mathrm{m}$ with pulse duration $18 \mathrm{~ns}$ and beam radius 1.6 $\mathrm{mm}$ was allowed to fall normally on the CMBA sample cell, it was converted into green light of wavelength $0.538 \mu \mathrm{m}$. The SHG efficiency is compared with KDP. From SHG test, the SHG efficiency of the grown CMBA crystal was found to be nearly 7.5 times greater than KDP. The second order optical properties of organic crystals can be greatly enhanced by altering the molecular alignment.

\section{Micro-hardness measurement}

The mechanical property plays a vital role in device fabrication. In the present work, the Knoop hardness indentations were carried out on the grown crystal for the applied load of 25, 50, 75 and $100 \mathrm{~g}$. The indentation time was kept as $10 \mathrm{~s}$ for each load. The long diagonal length was used in calculating the Knoop hardness number using the formula $\mathrm{H}_{v}=(1.8544 P) / \mathrm{d}^{2}$, where $\mathrm{P}$ is the applied load in $\mathrm{gm}$ and $\mathrm{d}$ is the mean diagonal length in microns. A graph was plotted between hardness number $\left(\mathrm{H}_{\mathrm{v}}\right)$ and applied load $(\mathrm{g})$ as shown in Fig.8. It shows that hardness of grown crystal is high at lower loads and decreases for higher loads.

\section{Conclusions}

Single crystals of 4-chloro-4-methyl benzylidene aniline were grown from ethanol by slow evaporation of the solvent at room temperature. Powder XRD confirms the crystallinity of the sample. The functional groups of the compound have been determined from FTIR and NMR spectrums. Optical absorption studies show that the sample is optically transparent over a wide wavelength region and minimum absorption is observed in the entire visible region. The melting point is found to be $108^{\circ} \mathrm{C}$ from the DSC curve. The SHG efficiency of CMBA is
Vol. 4 No. 6 (June 2011)

ISSN: 0974- 6846

found to be very high when compared with KDP. Knoop hardness tests shows that the crystal is stable up to $50 \mathrm{~g}$.

References

1. Prasad PN and Williams DJ (1991) Introduction to nonlinear optical effects in organic molecules and polymers. Wiley, NY.

2. Chemla DS and Zyss J (1987) Nonlinear optical properties of organic molecules and crystals. Academic Press, NY.

3. Bailey RT, Bourhill G, Cruickshank FR, Pug D, Sherwood JN and Simpson GS (1993) The linear and non linear optical properties of the organic non linear material 4 - nitro - 4' - methylbenzylidene aniline. J. Appl. Phys. 73 (4), 1591.

4. Lakshmana Perumal CK, Arulchakkaravarthi A, Rajesh NP, Santhana Raghvan P, Huang YC, Ichimura $M$ and Ramasamy $P$ (2002) Synthesis, crystal growth and FTIR, NMR, SHG studies of 4 Methoxy benzaldehyde - n- methyl - 4 - stilbazolium tosylate. J. Crystal Growth. 240, 212-217.

5. Yang W.Yu L, Zhang TL, Zhang JG, Ren FL, Liu YH, Wu RF and Guo JY (2006) Crystal structure and geometry-optimization study of 4-methyl-3',5'-dinitro4'-methyl benzylidene aniline. J. Mol. Struct. 794, 255- 260

6. Srinivasan K, Biravaganesh R, Gandhimathi $\mathrm{R}$ and Ramasamy P (2002) Growth \& Characterisation of NMBA single Crystals. J. Cryst. Growth. 236, 381392.

7. John O. Morley and Stuart D. Whittaker (2006) Non linear optical properties of thienylmethylene anilines and benzylidene aminothiophenes. J. Molecular Structure: THEOCHEM. 760, 1-13.

8. Nixon Azariah A, Haja Hameed AS, Thenappan T, Noel M and Ravi G (2004) Crystal growth and characterization of 4-nitro-4'- methoxy benzylidene aniline. Mater. Chem. Phy. 88, 90-96.

9. Leela S, Ramamurthi K and Bhagavannarayana G (2009) Synthesis, growth, spectral, thermal, mechanical and optical properties of 4-chloro- 4 dimethylamino- benzylidene aniline crystal; A third order non linear optical material. Spectrochimica Acta part A. 74, 78-83.

10. Shepherd EEA, Sherwood JN, Simpson GS and Yoon CS (1991) The growth, perfection and properties of organic non-linear optical materials I.4-nitro-4'-methyl benzylideneaniline. J. Crystal Growth, 113, 360-370.
Research article

CIndian Society for Education and Environment (iSee)
"Single crystal growth of organic NLO" http://www.indjst.org
Jothi \& Ramamurthi Indian J.Sci.Technol. 